\title{
Plasminogen Interaction with Trypanosoma cruzi
}

\author{
Laura Almeida , Gilmer Vanegas, Marina Calcagno, Juan Luis Concepción, Luisana Avilan ${ }^{+}$
}

\author{
Departamento de Biología, Facultad de Ciencias, Universidad de Los Andes, Mérida 5101, Venezuela
}

The ability of Trypanosoma cruzi to interact with plasminogen, the zimogenic form of the blood serin protease plasmin, was examined. Immunohistochemistry studies revealed that both forms, epimastigotes and metacyclic trypomastigotes, were able to fix plasminogen in a lysine dependant manner. This interaction was corroborated by plasminogen activation studies. Both forms of the parasite enhanced the plasminogen activation by tissue-type plasminogen activator. The maximal enhancements obtained were 15-fold and 3.4-fold with epimastigotes and metacyclic trypomastigotes, respectively, as compared to plasminogen activation in absence of cells. Ligand-blotting analysis of proteins extracted with Triton X-114 from a microsomal fraction of epimastigotes revealed at least five soluble proteins and one hydrophobic protein able to bind plasminogen.

Key words: Trypanosoma cruzi - plasminogen - plasminogen activation

Trypanosoma cruzi is the causative agent of Chagas disease, a public health problem in many countries of Latin America. During its life cycle, this parasite alternates between different morphological types, the epimastigote and the metacyclic trypomastigote in the insect vector and the amastigote and the bloodstream trypomastigote form in the mammal host. In the transmission from the vector to the mammal host, the parasite should transverse barriers such the extracellular matrix and reach susceptible host cells. Surface or secreted proteases have been implicated in parasite infections, among other functions, by their role in degrading proteins of the extracellular matrix (Santana et al. 1997). Additional surface protease activity for migration and spreading can be also acquired through the interaction with host proteins which do not stimulate the host's immune response. This additional activity could replace or supplement the parasite proteases. An increasing number of pathogens have the ability to bind plasminogen, a plasma and extracellular protein that is transformed, through the action of a plasminogen activator, in the broad-spectrum protease plasmin (Boyle \& Lottenberg 1997, Coleman \& Benach 1999). Moreover, several pathogens such as group A Streptococci, Staphylococcus aureus and Yersinia pestis, generate their own plasminogen activator (Lähteenmäki et al. 2001). Other plasminogen-binding pathogens may be capable of using host plasminogen activator (Lähteenmäki et al. 2001). In the case of Y. pestis, Borrelia burgdorferi, and group A Streptococci, the interaction with plasminogen has been demonstrated to be implicated in the invasiveness within the host (Gebbia et al. 1999, Li et al. 1999, Goguen et al. 2000). The suggested functions for plasmin in pathogenic infection include extracellular matrix protein degradation, fi-

Financial support: CDCHT-Universidad de los Andes, grant C10370003B and FONACIT, grant S12001000360

${ }^{+}$Corresponding author. Fax: +58-274-240.1286. E-mail: avilan@ula.ve

Received 14 July 2003

Accepted 10 December 2003 brin degradation, release of peptides for nutrition, and metalloprotease activation. Since the ability to interact with plasminogen has been shown in a trypanosomatid, Leishmania mexicana (Avilan et al. 2000, Calcagno et al. 2002), we explored, in this work, the plasminogen binding ability of $T$. cruzi.

\section{MATERIALS AND METHODS}

Parasites - T. cruzi EP strain were used in this work. Epimastigote were grown in liver-infusion tryptose (LIT) medium, supplemented with $10 \%$ heat inactivated fetal bovine serum, at $28^{\circ} \mathrm{C}$. Axenically derived metacyclic trypomastigotes were obtained by transferring epimastigotes in a defined medium TAU3AAG at $28^{\circ} \mathrm{C}$ (Contreras et al. 1994) following 3 day incubation. Metacyclic trypomastigotes were further purified using a DEAE-cellulose column as described elsewhere (De Souza 1983). Epimastigote forms used to obtain metacyclic trypomastigotes were maintained, for at least one year, by serial passage in LIT medium after being obtained from one mouse with parasitaemia.

Proteins - Human plasminogen was purified to homogeneity from fresh human plasma (Deutsch \& Mertz 1970). Tissue-type plasminogen activator (t-PA) was obtained from Chromogenix, Milano, Italy. The following antibodies were used for immunohistochemical studies: goat antihuman plasminogen (Calbiochem, San Diego, US) and rabbit anti-goat IgG conjugated with peroxidase (Sigma, St. Louis, US).

Plasminogen immunoperoxidase staining - Living parasites $\left(\sim 1 \times 10^{7}\right.$ cells $)$ obtained under different specified conditions were incubated with $2 \mu \mathrm{M}$ plasminogen in phosphate buffered saline (PBS), $\mathrm{pH} 7.4$, containing $0.1 \%$ BSA (final volume $250 \mu \mathrm{l}$ ) for $90 \mathrm{~min}$ in the absence or the presence of $10 \mathrm{mM} \varepsilon$-aminocaproic acid. At the end of the incubation period, the cells were pelleted, washed three times with $2 \mathrm{ml}$ PBS and resuspended in the same buffer. Parasites suspension were placed onto glass slides, air dried, and fixed with acetone. Fixed parasites were sequentially incubated with normal rabbit serum, diluted 1:80, the primary goat anti-human plasminogen antibodies at 1:640 dilution, and peroxidase conjugated rabbit anti-goat 
IgG secondary antibodies at 1:400 dilution. A 3-amino-9ethyl-carbazole- $\mathrm{H}_{2} \mathrm{O}_{2}$ substrate was used to visualize the immunoreactivity. Cells treated with normal serum and/or with the secondary antibodies alone, were used as controls. The parasite staining was evaluated by light microscopic observations.

Plasminogen activation assays - Plasminogen activation rate was performed by monitoring the amidolytic activity of generated plasmin in a test volume of $100 \mu$ l. Plasminogen $(200 \mathrm{nM})$ was incubated at room temperature in PBS (final volume $100 \mu \mathrm{l}$ ) containing $0.6 \mathrm{mM}$ plasmin substrate S2251 (Chromogenix) in the presence or absence of parasites. Activation of plasminogen was initiated by addition of plasminogen activator $(10 \mathrm{nM}$ tissue-type plasminogen activator, t-PA). The hydrolysis of the chromogenic substrate was monitored continuously by measuring the absorbance at $405 \mathrm{~nm}$. Initial velocities were calculated from the slope of plots $\mathrm{A}_{405 \mathrm{~nm}}$ versus $\mathrm{t}^{2}$ as described elsewhere (Wohl et al. 1980, Stack et al. 1991).

Triton X-114 phase - Microsomal fraction were obtained by differential centrifugation from $T$. cruzi epimastigotes cultured in two liters of LIT medium as described previously (Caceres et al. 2003). A stock of $10 \%$ Triton X-114 was added to this microsomal fraction to a final concentration of $2 \%$ Triton X-114. Phase partition was carried out incubating the sample at $-20^{\circ} \mathrm{C}$ overnight and then at $28^{\circ} \mathrm{C}$ for $10 \mathrm{~min}$. Detergent (lower) and aqueous (upper) phases were recovered and the proteins precipitated with cold acetone.

Ligand blotting - Microsomal proteins (soluble and hydrophobic) obtained after Triton X-114 extraction were separated by SDS-polyacrylamide electrophoresis as described by Laemmli (1970) under non-reducing condition, and parallel gels were either stained with Coomasie blue or transferred to nitrocellulose membrane as described elsewhere (Sambrook et al. 1989). For ligand-blotting, membranes were washed in PBS and blocked overnight with PBS containing $2 \%$ gelatin. The membranes were then probed with $2 \mu \mathrm{M}$ plasminogen in PBS-gelatin for $1 \mathrm{~h}$, in the presence of $2 \mathrm{mM}$ phenylmethanesulphonyl fluoride (PMSF) and 2mM EDTA. In some experiments the incubation solution contained $20 \mathrm{mM} \varepsilon$-aminocaproic acid. After three washings, the membrane was incubated with antihuman plasminogen antibody for an additional $1 \mathrm{~h}$. Thereafter, the membrane was rinsed with PBS and incubated with the alkaline phosphatase-conjugated second antibody and revealed with 5-bromo-4-chloro-3-indolyl phosphate/nitro blue tetrazolium (BCIP/NBT) as substrate.

\section{RESULTS AND DISCUSSION}

A direct evidence of plasminogen binding to T. cruzi is provided by immunostaining of plasminogen-treated parasites (Fig. 1). Both metacyclics trypomastigotes and epimastigotes resulted positive to plasminogen binding (Fig. 1B, 1E). Moreover, in both cases, the staining was reduced by the presence, in the incubation mixture, of $\varepsilon$ aminocaproic acid, a lysine analogue (Fig. 1C, 1F). These results suggest that the lysine binding kringle structure, homologous triple-loop structures (Ponting et al. 1992), of the plasminogen molecule might be involved in the association of plasminogen with the parasite. These lysine binding sites have been implicated in the interaction of plasminogen with fibrin and with surface proteins on a variety of cells including pathogen organisms (AnglesCano 1994, Lähteenmäki et al. 2001). Plasminogen staining was visualized in parasites incubated in TAU3AAG medium during several days. However, the staining resulted weak with seven days of incubation (data not shown).

When plasminogen interacts with molecules such as receptors or fibrin, a conformational change is produced that permit an enhancement of plasminogen activation by physiological activators (Angles-Cano 1994). To corroborate the plasminogen binding onto the T. cruzi surface, we investigated the plasminogen activation by t-PA in the presence of different cell concentrations. The experiments were performed with either epimastigotes or metacyclic trypomastigotes. As shown in Fig 2, both types of forms were able to facilitate the plasminogen activation by t-PA in cell concentration-dependent manner, the epimastigote form being more effective in producing the plasminogen activation enhancement. The maximal enhancements obtained were $15 \pm 3.2$ and $3.4 \pm 1$ for epimastigotes and metacyclic trypomastigotes, respectively, compared to plasminogen activation in absence of cells. No plasmin activity was observed when plasminogen activator was omitted (data not shown) indicating that $T$. cruzi cells do not produce a plasminogen activator. The plasminogen activation enhancement by $T$. cruzi shows that this parasite is able to bind plasminogen on its surface and that $T$. cruzi can act as a regulator of this activation. The lower enhancement of plasminogen activation by metacyclic trypomastigotes compared to epimastigotes could reflect the molecular changes associated to culture conditions. Axenic metacyclic forms derived from epimastigotes maintained more than 78 days in culture are less virulent and have different protein profile than the insect-born metacyclic trypomastigotes (Contreras et al. 1998). By contrast, the protein profile of epimastigotes do not change by several passage in axenic medium (Contreras et al. 1998).

Finally, an attempt to identify $T$. cruzi plasminogen receptor was performed using a ligand-blotting assay on proteins from a microsomal fraction extracted with Triton $\mathrm{X}-114$. The treatment with this detergent allows to separate hydrophilic proteins (aqueous phase) and membrane bound proteins (detergent phase). This assay allowed the demonstration of at least five plasminogen binding proteins in the soluble fraction, of $110,37,32,19$, and $13 \mathrm{kDa}$ of molecular masses and one plasminogen binding protein of $15 \mathrm{kDa}$ of molecular mass in the detergent fraction (Fig. 3A). Control blotting in which plasminogen was omitted showed no band reactivity (data not shown). Incubation of membranes with plasminogen and $\varepsilon$ aminocaproic acid abolished the binding to the parasite proteins, indicating specific binding through lysine binding sites in the plasminogen molecule (Fig. 3B). Several proteins have been identified as plasminogen receptors in numerous cell types such as vertebrate cells and pathogenic organisms (Plow et al. 1991, Lähteenmäki et al. 2001, Crowe et al. 2003). Moreover, in Candida albicans, at least six plasminogen binding proteins were found (Crowe 
et al. 2003). These receptors are characterized by a relative low affinity for plasminogen. The identification of the plasminogen binding protein in T. cruzi will allow determining whether it is structurally related to other proteins which have been identified as plasminogen receptor.

The interaction with plasminogen may be important in the establishment of the infection into the vertebrate host where the plasminogen is available. Plasminogen activated on the parasite surface could contribute to cross barriers for migration. The fact that metacyclic trypomastigote is able to bind plasminogen is consistent with this hypoth- esis. However, in this study we found that epimastigote, a form that is exclusively found in the vector host, is also able to interact with plasminogen. Moreover, this form produced a higher enhancement of plasminogen activation than the axenic metacyclic trypomastigote. One may speculate that plasminogen-binding molecules could have additional functions in the parasites and for that the plasminogen binding property may be found in vitro in either epimastigotes or in metacyclic trypomastigote. Different functions for plasminogen receptors have been found in pathogens. In group A streptococci, the two active en-
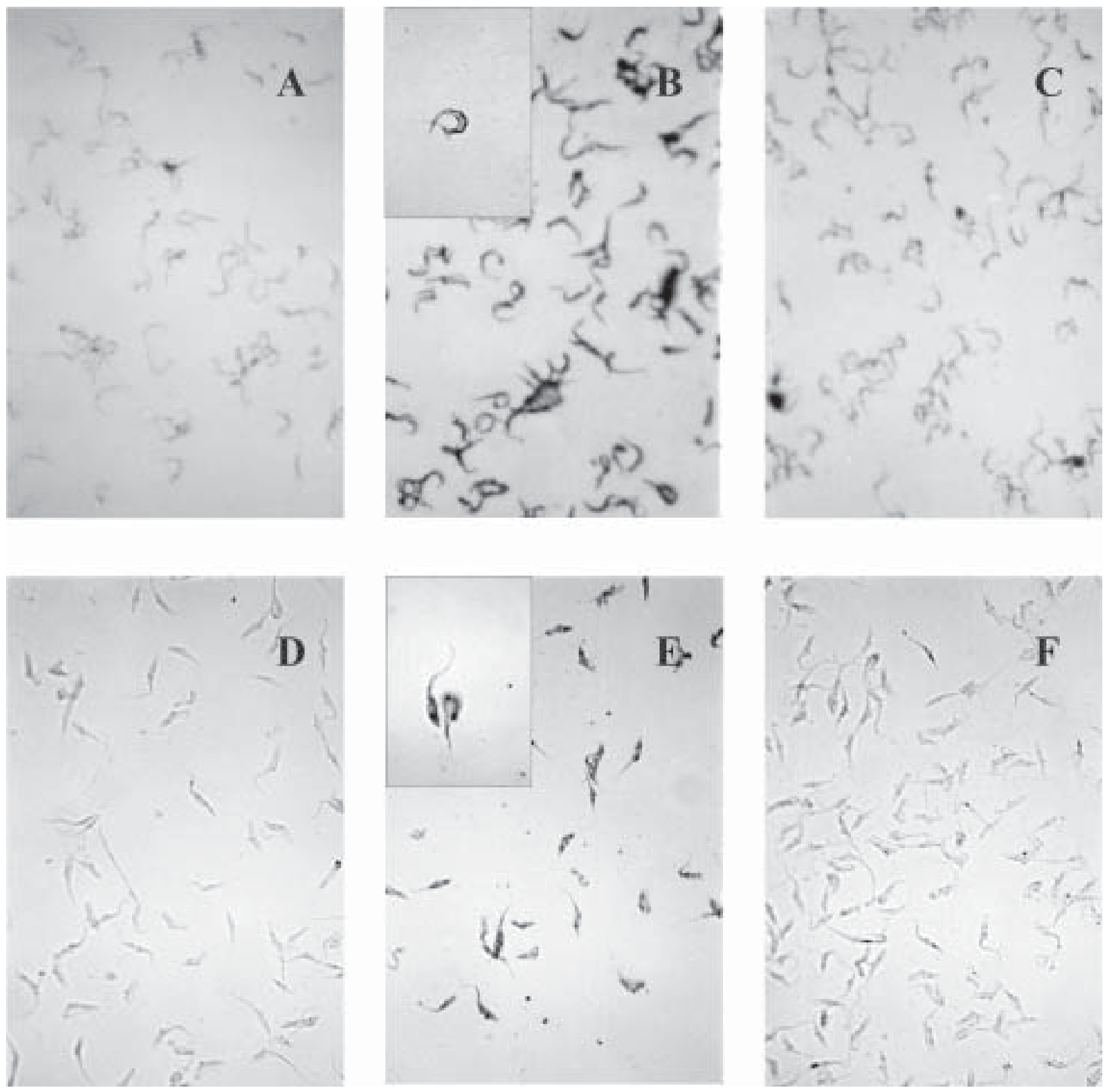

Fig. 1: plasminogen immunostaining of plasminogen treated Trypanosoma cruzi. Living parasites were incubated with $2 \mu \mathrm{M}$ plasminogen in the presence or absence of $\varepsilon$-aminocaproic acid. The slides were sequentially incubated with anti human plasminogen antibody and a peroxidase conjugated secondary antibody. A: metacyclic trypomastigotes without plasminogen treatment; B: plasminogen treated metacyclic trypomastigotes; C: metacyclic trypomastigotes with plasminogen treatment in the presence of $\varepsilon$-aminocaproic acid; D: epimastigotes without plasminogen treatment; E: plasminogen treated epimastigotes; F: epimastigotes with plasminogen treatment in the presence of $\varepsilon$ aminocaproic acid. The magnification was X650. Insets in B and E, plasminogen treated parasites, magnification X2500. 

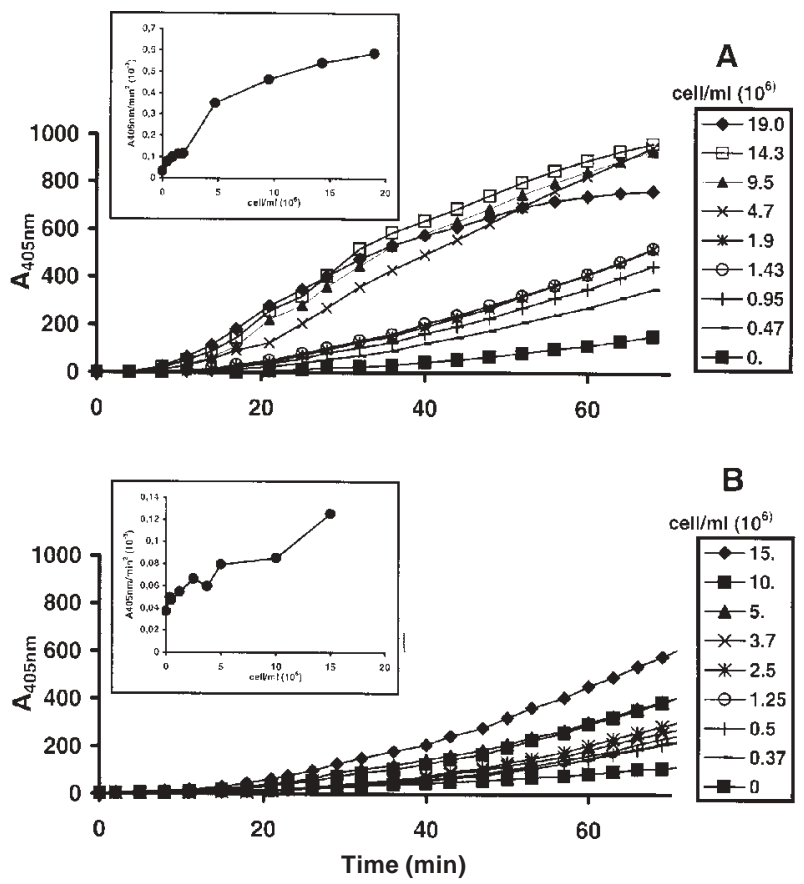

Fig. 2: activation of plasminogen in the presence of Trypanosoma cruzi. $10 \mathrm{nM}$ t-PA was added to a mixture, in a final volume of 100 $\mu$, containing $200 \mathrm{nM}$ plasminogen, $0.6 \mathrm{nM} \mathrm{S2251}$ and different concentrations of epimastigotes (A) and metacyclic trypomastigotes (B). The absorbance at $405 \mathrm{~nm}$, at different times, was determined. Insets, effect of cell density on t-PA-catalyzed plasminogen activation.
A

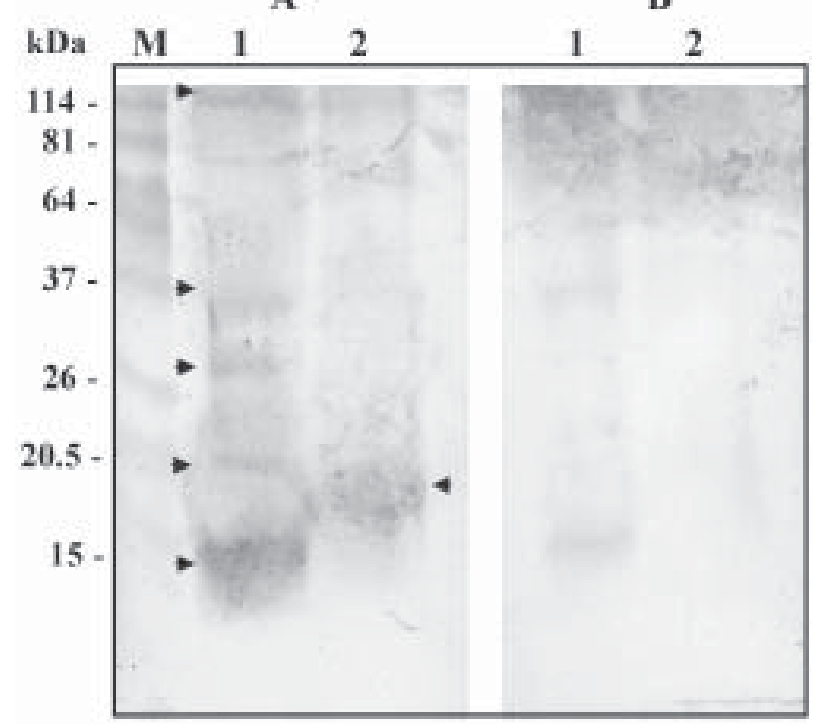

Fig. 3: ligand blotting. Proteins from Triton X-114 extraction of microsomal fraction of Trypanosoma cruzi epimastigotes were submitted to SDS-PAGE, under non-reducing conditions, transferred to nitrocellulose and incubated with $2 \mu \mathrm{M}$ plasminogen. Bound plasminogen was revealed with anti-human plasminogen antibody and phosphatase alkaline-second antibody. A: Blot incubated with plasminogen; B: Blot incubated with plasminogen and $20 \mathrm{mM} \varepsilon$ aminocaproic acid. Lane 1: soluble proteins. Lane 2: hydrofobic proteins. M: molecular weight markers. Arrows indicate the electrophoretic mobility of plasminogen binding proteins. zymes, glyceraldehyde 3-phosphate dehydrogenase and $\alpha$-enolase, both found on cell surface, are also plasminogen binding molecules (Lähteenmäki et al. 2001). Another possible explanation for the presence of plasminogen receptor in epimastigote is that plasminogen provided by the blood meal can have some function in the vector. Such is the case of B. burdorferi, in which the plasminogen interaction is important for the establishment in the vector (Coleman et al. 1997). To know whether the plasminogen interaction with $T$. cruzi has a physiological function in either the vertebrate host or the insect vector, in vivo studies are required such as experimental infection in plasminogen-deficient transgenic mice and vector-feeding experiments.

\section{ACKNOWLEDGMENTS}

To Dr Victor Contreras for help with the metacyclic trypomastigote culture.

\section{REFERENCES}

Anglés-Cano E 1994. Overview on fibrinolysis: plasminogen activation pathways on fibrin and cell surfaces. Chem Phys Lipids 67/68: 353-362.

Avilan L, Calcagno M, Figuera M, Lemus L, Puig J, Rodriguez AM 2000. Interaction of Leishmania mexicana promastigotes with the plasminogen-plasmin system. Mol Biochem Parasitol 110: 183-193.

Boyle MDP, Lottenberg R 1997. Plasminogen activation by invasive human pathogens. Thromb Haemost 77: 1-10.

Caceres AJ, Portillo R, Acosta H, Rosales D, Quiñones W, Avilan L, Salazar L, Dubourdieu M, Michels PAM, Concepcion JL 2003. Molecular and biochemical characterization of hexokinase from Trypanosoma cruzi. Mol Biochem Parasitol 126: 251-262.

Calcagno M, Avilan L, Colasante C, Berrueta L, Salmen S 2002. Interaction of different Leishmania mexicana morphotypes with plasminogen. Parasitol Res 88: 972-978.

Coleman JL, Benach JL 1999. Use of plasminogen activation system by microorganisms. J Lab Clin Med 134: 567-576.

Coleman JL, Gebbia JA, Piesman J, Degen JL, Bugge TH, Benach JL 1997. Plasminogen is required for efficient dissemination of $B$. burgdorferi in ticks and for enhancement of spirochetemia in mice. Cell 89: 1111-1119.

Contreras VT, Araque W, Delgado VS 1994. Trypanosoma cruzi: metacyclogenesis in vitro - I. Changes in the properties of metacyclic trypomastigotes maintained in the laboratory by different methods. Mem Inst Oswaldo Cruz 89: 253259.

Contreras VT, De Lima AR, Zorrilla G 1998. Trypanosoma cruzi: maintenance in culture modify gene and antigenic expression of metacyclic trypomastigotes. Mem Inst Oswaldo Cruz 93: 753-760.

Crowe JD, Sievwright IK, Auld GC, Moore NR, Gow NAR, Booth NA 2003. Candida albicans binds human plasminogen: identification of eight plasminogen-binding proteins. Mol Microbiol 47: 1637-1651.

De Sousa MA 1983. A simple method to purify biologically and antigenically preserved bloodstream trypomastigotes of Trypanosoma cruzi using DEAE-cellulose columns. Mem Inst Oswaldo Cruz 78: 317-333.

Deutsch DG, Mertz ET 1970. Plasminogen: purification from human plasma by affinity chromatography. Science 170: 1095-1096.

Gebbia JA, Monco JCG, Degen JL, Bugge TH, Benach JL 1999. The plasminogen activation system enhances brain and heart 
invasion in murine relapsing fever borreliosis. J Clin Invest 103: 81-87.

Goguen J, Bugge T, Degen JL 2000. Role of the pleiotropic effects of plasminogen deficiency in infection experiments with plasminogen-deficient mice. Methods 21: 179-183.

Laemmli UK 1970. Cleavage of structural proteins during the assembly of the head of bacteriophage T4. Nature 227: 680-685.

Lähteenmäki K, Kuusela P, Korhonen TK 2001. Bacterial plasminogen activators and receptors. FEMS Microbiol Rev 25: 531-552.

Li Z, Ploplis VA, French EL, Boyle MDP 1999. Interaction between group A streptococci and the plasmin(ogen) system promotes virulence in a mouse skin infection model. J Infect Dis 179: 907-914.

Plow EF, Felez J, Miles LA 1991. Cellular regulation of fibrin- olysis. Thromb Haemost 66: 32-36.

Ponting CP, Marshall JM, Cederholm-Williams SA 1992. Plasminogen: a structural review. Blood Coagul Fibrinolysis 3: 605-614.

Sambrook J, Fritsch EF, Maniatis T 1989. Molecular Cloning: A Laboratory Manual, 2nd ed., Cold Spring Harbor Laboratory Press, Cold Spring Harbor, NY.

Santana JM, Grellier P, Schrével J, Teixeira ARL 1997. A Trypanosoma cruzi-secreted $80 \mathrm{kDa}$ proteinase with specificity for human collagen types I and IV. Biochem J 324: 129-137.

Stack S, Gonzalez-Gronow M, Pizzo SV 1991. The effect of divalent cations on the conformation and function of human plasminogen. Arch Biochem Biophys 284: 58-62.

Wohl RC, Summaria L, Robbins KC 1980. Kinetics of activation of human plasminogen by different activator species at pH 7.4 and $37^{\circ} \mathrm{C}$. J Biol Chem 255: 2005-2013. 